\title{
Malagasy dialects in Mayotte
}

\author{
MAURIZIO SERVA \& MICHELE PASQUINI
}

January 9, 2021

Dipartimento di Ingegneria e Scienze dell'Informazione e Matematica, Università dell'Aquila, L'Aquila, Italy.

\begin{abstract}
The Malagasy language is not strictly confined to Madagascar but it is also spoken in Mayotte, one of the four islands which constitute the Comoros archipelago. The inhabitants of Mayotte are, in fact, divided between two very distinct mother languages, one is Shimaore, a Bantu dialect similar to the dialects spoken in the other three islands, the other is Malagasy, an Austronesian language. In turn, Malagasy is represented by two distinct varieties, one is the Kibosy Kimaore, a name which simply means "Malagasy of Mayotte" (this name is sometimes used to indistinctly denote both varieties) and the other is the Kibosy Kiantalaotsy.

It is usually stated that Kibosy Kimaore is close to the Northern Madagascar varieties, while Kibosy Kiantalaotsy is very close to Mahajanga Sakalava (Glottolog classifies Mahajanga Sakalava and Kibosy Kiantalaotsy as twin varieties). While we clearly confirm the cladistic position of Kibosy Kimaore, we find that Kibosy Kiantalaotsy is not particularly close to Mahajanga Sakalava, but it is closer to the Sakalava dialects of the South-West of Madagascar. Moreover, Kibosy Kiantalaotsy seems to be rather the result of multiple contributions from Madagascar than the offspring of a single dialect or a few dialects.

Our outcome is based upon the comparison of the two Mayotte varieties with sixty different Madagascar varieties.
\end{abstract}

Keywords: Malagasy dialects, Austronesian languages, taxonomy of languages, lexicostatistics, Malagasy varieties in Mayotte.

\section{Preamble and data-set}

Mayotte is the easternmost island of the Comoros archipelago which lies between the North-West of Madagascar and the South-Eastern coast of Africa. While the three other islands of the archipelago, Grande-Comore (Ngazidja), Mohéli (Mwali), Anjouan (Ndzuani), became independent in 1975, Mayotte (Maore) chose to become an overseas department of France. However, on a cultural and religious level, Mayotte is part of the same whole as the three other islands. Only on a strictly linguistic point of view there is a difference since a non negligible fraction of the inhabitants of Mayotte speaks a Malagasy language (locally named Kibosy) instead of a Bantu language. Malagasy speakers do not occupy a part of the island but they live in about twenty villages scattered all around, especially on the East coast. In no way they consider themselves as Malagasy people or they show peculiar differences from the other inhabitants of Mayotte.

Malagasy is an Austronesian language, totally distinct from Bantu languages. The Austronesian expansion, which very likely originated in the South of China and went through Taiwan, is probably the most spectacular event of maritime colonization in human history, Madagascar being the Western edge of this expansion. The colonizers from Indonesia probably landed around $650 C E$ in the South-East of Madagascar [1]. There is a huge literature on this subject in linguistics [2-9] and genetics [10-16] and a role of the Comoros archipelago is occasionally evoked. This role is implicitly excluded in [1] since a landing in the South-East of Madagascar is compatible with a direct navigation from Indonesia. Moreover, a team of archaeologists recently discovered that rice, mung beans and cotton cultivated in Madagascar and in Comoros have been introduced from Southeast Asia [18] which also testifies in favor of a direct navigation. However, it has to be emphasized Adelaar's 


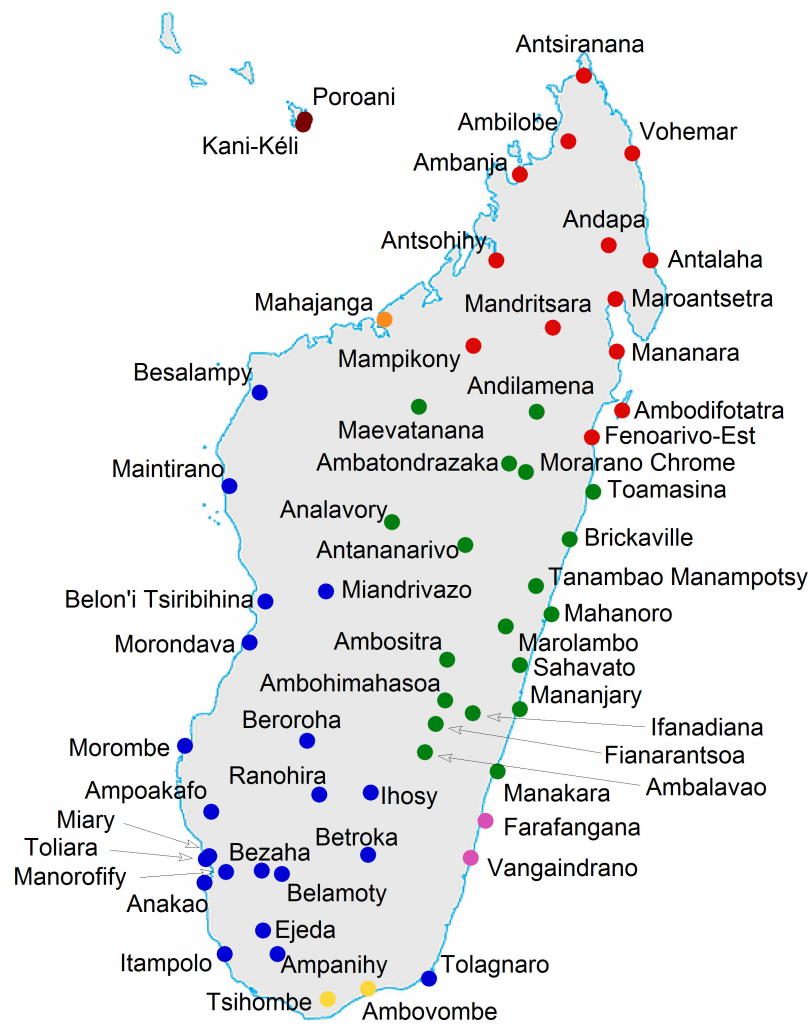

Figure 1: The map with the names of the towns/villages where the $60+2$ varieties were collected. The names of the ethnicities are missing, nevertheless they can be found in the Supporting Information (Table 1). The colors of the continental varieties correspond to the classification that we proposed in [1]. The main partition is red+orange+green on one branch versus blue+yellow+violet on the other. This implies that the Sakalava dialects of the West and South-West coast (blue spots from Besalampy to Toliara) are deeply different from Northern dialects (red+orange), included the Sakalava of Mahajanga (orange) and the Sakalava of Ambanja (red). Both in Toliara and in Morondava two different varieties coexist, which implies two pairs of coinciding spots. The two brown spots corresponding to the two Kibosy varieties in Mayotte also are almost coincident because the villages of Poroani and Kani-Kéli are geographically very close.

authoritative opinion [17] who reconciles this discovery with the hypothesis of an indirect navigation, with an intermediate stop on the East coast of Africa and possibly via the Comoros.

In all cases, the presence of Malagasy speakers in Mayotte seems to be the output of more recent events, not much older than a pair of centuries. In this paper we will try to not deeply enter into this storytelling and historical dating will be involved only when necessary.

Kibosy (Malagasy of Mayotte) is divided into two distinct varieties, one is the Kibosy Kimaore and the other is the Kibosy Kiantalaotsy. Although Kibosy is spoken in about twenty villages, the Kiantalaotsy variety is confined to only two of them: Ouangani and Poroani (and, eventually, Mtsapere, a suburb of Mamoudzou where it is spoken by a part of its inhabitants) $[19,20]$. In this paper, as representative of Kibosy Kiantalaotsy we consider the dialect spoken in Poroani (geodesic coordinates in decimal degrees: $-12.89^{\circ} S, 45.14^{\circ} E$ ), while the Kibosy Kimaore is represented by the dialect spoken in Kani-Kéli $\left(-12.96^{\circ} S, 45.11^{\circ} E\right)$.

The data-set we use for our research consists in 60 Swadesh lists of 207 items for the varieties of Madagascar (continental varieties or continental dialects hereafter) and 2 Swadesh lists of 207 items for the Mayotte's varieties. 
The 12,420 terms corresponding to the sixty continental dialects were collected by one of us (M.S.) during the years 2018 and 2019. Each list corresponds to a different variety, which is not simply identified by the name of the ethnicity but also by the location where the variety was collected. In turn, the location is identified by the name of a town/village and by latitude and longitude. Each list was furnished and checked at least by three native language speakers which, for each given meaning, were asked to furnish the most common word in their dialect as spoken in their town/village. The 60 lists can be found in the Supporting Information of paper [1].

Most of the 207 terms of Kimaore and part of the 207 terms of the Kiantalaotsy were drawn from Dictionnaire du dialecte malgache de Mayotte (Comores) by Noël J. Gueunier, édition revue et corrigée (2016), publshed by KomEDIT and cross-checked by consulting Webonary - Dictionaries and Grammars of the World, https://www.webonary.org/kibushi/. The Kiantalaotsy list was largely integrated and corrected by Professor Mlaïli Condro in Mayotte and by Lina Hijab a native Kiantalaotsy speaker. A few missing words of both lists were provided by various Poroani and Kani-Kéli inhabitants which also helped to verify that all terms correspond to the specific variety of their village. The 2 lists can be found in the Supporting Information of this paper (Dataset 1).

A complete overview of the geographical locations of the 62 dialects can be appreciated in Fig. 1, the colors of the 60 continental varieties correspond to the classification which we proposed in [1], while the color of the 2 Mayotte dialects is brown. Eventually, the precise latitude and longitude coordinates as well as the names of the corresponding towns/villages and ethnicities of all the 62 varieties can be found in the Supporting Information of this paper (Table 1).

\section{Genealogical distances}

A part of the information contained in the $N=62$ short vocabularies which constitute our data-set can be encoded into the $N(N-1) / 2=1891$ lexical distances between each pair of languages, which can be considered as the entries of a $N \times N$ upper triangular matrix. Obviously, a lot of information is lost in the procedure, but what remains is basically what is needed for the goals of this paper.

The idea of measuring the distance between two languages comparing short vocabularies seems to have its roots in the work of the French explorer Jules-Sébastien-César Dumont d'Urville which collected his comparative word lists during his voyages aboard the Astrolabe from 1826 to 1829 [21]. About 70 years ago Morris Swadesh [22] proposed a similar method using core vocabularies with 100 or 200 terms which are known as Swadesh lists. In the present work we employ an automated refined version of the Swadesh approach proposed by Serva and Petroni about ten years ago [23].

As already mentioned, all our vocabularies consist in lists of 207 words associated to the same 207 meanings. Comparing the two lists which correspond to a pair of languages (say language $\alpha$ and language $\beta$ ), it is possible to determine their lexical distance $D(\alpha, \beta)$ which is a number between 0 (languages are identical) and 1 (languages are totally different)

Once a lexical distance $D(\alpha, \beta)$ of two contemporary languages has been computed from the lists, it is possible to transform it in a genealogical distance $T(\alpha, \beta)$, which is the time from the last common ancestor language. The fundamental formula of Glottochronology states that

$$
T(\alpha, \beta)=-\frac{\tau}{2} \ln [1-D(\alpha, \beta)]
$$

where the characteristic time $\tau$ is the inverse of the replacement rate. In the idea of Swadesh $\tau$ should have been a universal constant, but this is not the case [24], [25], which, among other things, entails that some languages had and have a slower (or faster) evolution than others.

Indeed, the value of $\tau$ is irrelevant in this paper, notwithstanding, for comparison purposes, we set $\tau=5136$ years, which is the value that we found in [1] for the Malagasy family (meaning the sixty continental varieties). This value is substantially meaningless when referred to distances involving the two Mayotte dialects because of the strong distortion due to the important horizontal transfer from the local Comorian Bantu (Shimaore).

In Fig. 2 we have plotted the genealogical distances (in kiloyears) of the continental varieties from Kibosy Kimaore (left) and from Kibosy Kiantalaotsy (right). In both cases we have considered only the first twenty smallest distances which are ranked in an increasing order. Neither Kibosy Kimaore nor Kibosy Kiantalaotsy are particularly close to Mahajanga Sakalava which for both dialects is barely in the twelfth position in ranking. 

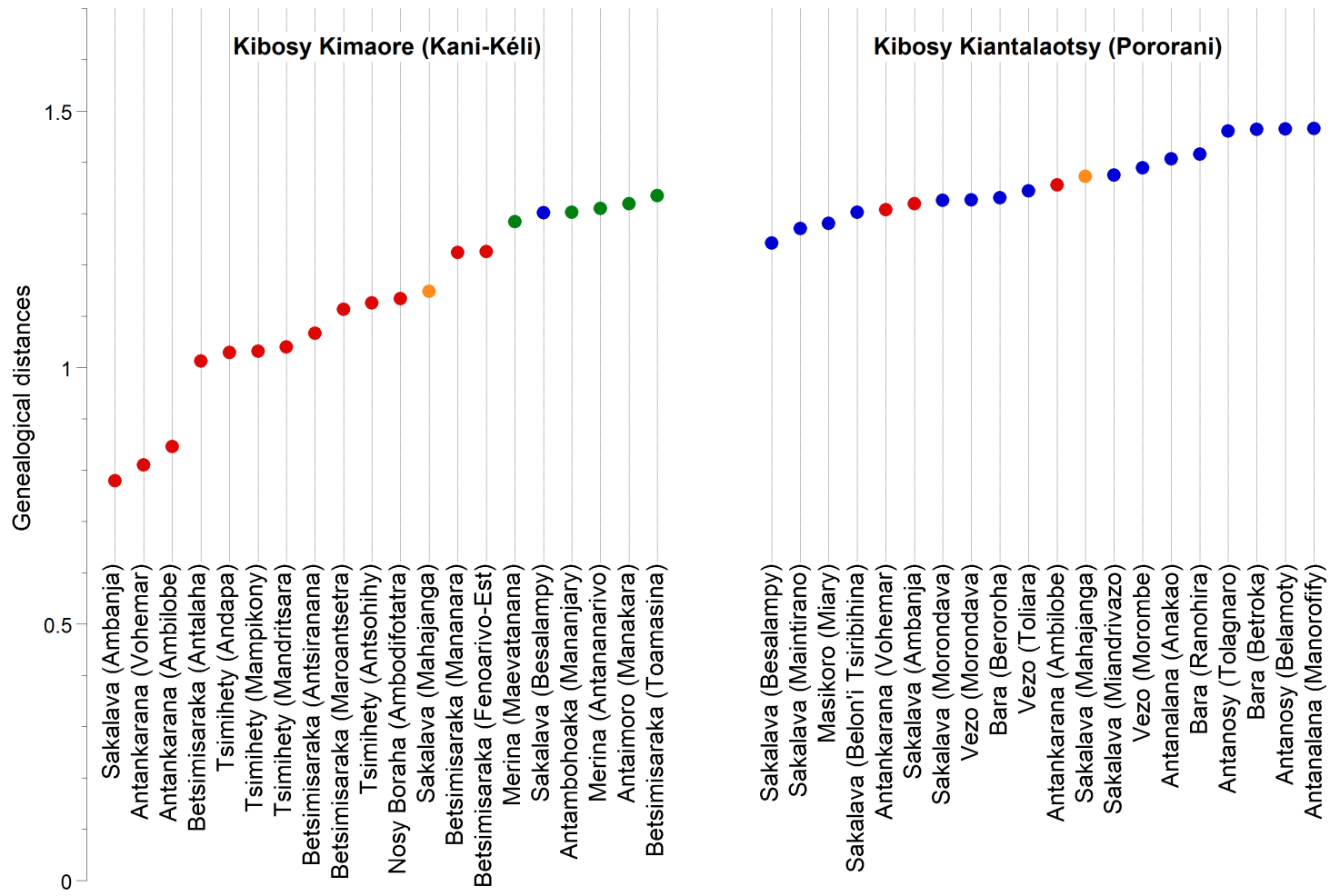

Figure 2: Ranked genealogical distances (in kiloyears) of the continental varieties from Kimaore and from Kiantalaotsy (in both cases only the first 20 smallest distances are plotted). These first twenty ranked varieties are predominantly Northern dialects for Kimaore and South-Western dialects for Kiantalaotsy. Moreover, for Kimaore all the closest ten varieties are from North (red spots), while for Kiantalaotsy eight of the closest ten are from South-West (blue spots), but two of them are from North. Concerning Kimaore, the Sakalava of Ambanja (Nosy Be region) is the variety at the minimal genealogial distance while, concerning Kiantalaotsy, it is the Sakalava of Besalampy. Neither Kimaore nor Kiantalaotsy are particularly close to Mahajanga Sakalava (orange spot). It should be noted that the distances from Kiantalaotsy vary in a small range (many dialect are almost equally close) compared to the Kimaore ones (a few dialects are much closer than the others).

This outcome contrasts the common wisdom that Kibosy Kiantalaotsy is extremely close to Mahajanga Sakalava (Glottolog classifies Mahajanga Sakalava and Kibosy Kiantalaotsy as twin varieties). Moreover, in any possible genealogical tree construction, Kiantalaotsy lies far away from Mahajanga Sakalava. This is not simply due to a difference between the true phylogeny and a possibly biased cladistic generated by lexical distances whose values are influenced by non-philogenetic factors. When genealogy exists, it is usually unveiled by standard tools based on vocabularies. This is evident if one considers the position of English in the genealogical tree of Indo-European languages in Serva and Petroni [23]: despite the relatively small distance from French, English is rightly placed in the Germanic branch.

It should be noted that for Kimaore all the closest ten varieties are from the North of Madagascar (red spots), which clearly implies an origin in that region. On the contrary, for Kiantalaotsy eight of the closest ten are from the South-West (blue spots), but two Northern varieties are also present in the fifth and sixth position. This Northern presence may be the consequence of the local horizontal transfers from Kimaore, which has much more speakers or Kiantalaotsy or it may may be a first clue to a plural origin of this last dialect. However, even if Kiantalaotsy also exhibits contributions from Northern Sakalava and Antankarana varieties, its vocabulary has a South-Western Sakalava main core, since most of its closest relatives are South-Western Sakalava dialects, included Vezo and Masikoro. Similar conclusion, concerning this specific point were reached by Gueunier [19]. 
It should be also noted that the distances from Kiantalaotsy vary in a small range (many dialects are almost equally close) compared to the Kimaore ones (a few dialects are much closer than the others). This fact confirms that Kimaore has a clear origin in the North while it provides more evidence to the hypothesis that Kiantalaotsy has a mixed origin.

In sum, we clearly confirm the cladistic position of Kibosy Kimaore as an offspring of Northern dialects, the closest continenta variety being Ambanja Sakalava (Nosy Be area). This scenario is compatible with the 1832 conquest of Mayotte sultanate by Andriantsoly, a former Iboina King, who reached Mayotte after having passed through Nosy Be where he probably collected his crew. At that time the population of Mayotte was very small, probably 3.000 individuals included the Mozambican slaves, as a consequence of a previous period of troubles and piracy (1790-1820) mostly leaded by Malagasy sailors [19], therefore even a small group of Northern Malagasy immigrants could be at the origin of the whole modern Kibosy Kimaore population.

On the contrary, the different origin of Kibosy Kiantalaotsy is not clear. We found that it is not particularly close to Mahajanga Sakalava, on the contrary, it seems to be close to the South-Western Sakalava dialects of Madagascar (Besalampy and Maintirano Sakalavas are the closest). Nevertheless, many other Sakalava dialects from South-West and also two from North seem to have contributed in almost equal proportion to this language (their genealogical distance are almost the same as it can be seen in the right panel of Fig. 2). This feature is probably a consequence of the fact that this language originated by commercial contacts leaded by Antalaotse, muslim traders scattered along all the West coast of Madagascar [4]. The presence of an Antalaotse outpost of in Mayotte is attested at the very beginning of the XIXth century [20] or even before. Therefore, Kiantalaotsy is probably the oldest Malagasy variant in Mayotte. This older presence is confirmed by the fact that we measured a smaller genealogical distance between Kiantalaotsy and Shimaore (4.16 kiloyears) with respect to the distance between Kimaore and Shimaore (4.45 kiloyears). This means a larger amount of horizontal transfers between Kiantalaotsy and Shimaore, likely due to the longer contact between these two varieties.

Another possibility, which we find less likely, is that Kiantalaotsy speakers are the descendants of a much older Malagasy population. Malagasy cultural markers were confirmed as early as the XIth century, with the presence of an Austronesian-type furnace at Bagamoyo [26]. This presence may have endured in Mayotte among the Kiantalaotsy speakers.

Finally we would like to mention that the genealogical distance between Kimaore and Kiantalaotsy is quite high (0.98 kiloyears), many of the distances between continental varieties are smaller and also the distance between Kimaore and Ambanja Sakalava (0.78 kiloyears) is smaller. This large distance, which has survived to a pair of centuries of contact in Mayotte, testifies a well different origin for the two varieties.

\section{Reconstructed geography}

The preliminary analysis in the previous section shows that the relations between the two Mayotte dialects and the continental varieties cannot be interpreted in terms of pure phylogenesis because the makeup of at least one of them seems to be the consequence of multiple contacts and/or multiple origins. In fact, when we tried to construct phylogenetic trees in order to clarify the Kiantalaotsy cladistic position, we didn't succeed. This dialect stands on a separate branch because it is almost equally lexically distant from various continental varieties and it hasn't a privileged link to any of them.

Although phylogenetic descriptions are ubiquitous in linguistics, they give an incomplete and sometimes inadequate representation of the information contained in the matrix of genealogical distances. The main reason of inadequacy lies in the horizontal transfer process between geographically close languages (vocabulary borrowings). This is especially true when a family of languages represents a continuum both geographically and linguistically as the Malagasy family of varieties. In this case, horizontal transfers turn out to be the primary aspect of the dynamics of the languages [24]. Moreover, since geographically close dialects exchange vocabulary more easily than those which are far away, this phenomenon weaves a lexical affinity network which connects all of them [27]. Finally, phylogenetic descriptions obviously fail in the infrequent cases where a straight phylogenesis does not exist at all.

In order to grasp the information conveyed by horizontal transfers between continental varieties and to clarify the position of Kiantalaotsy with respect to them, we adapt the method proposed in [1], which, in turn, is an implementation of the procedure in [27]. This method allows to construct the purely lexical geography of Madagascar (plus Mayotte) by the matrix of genealogical distances. It is very important to be aware that this 

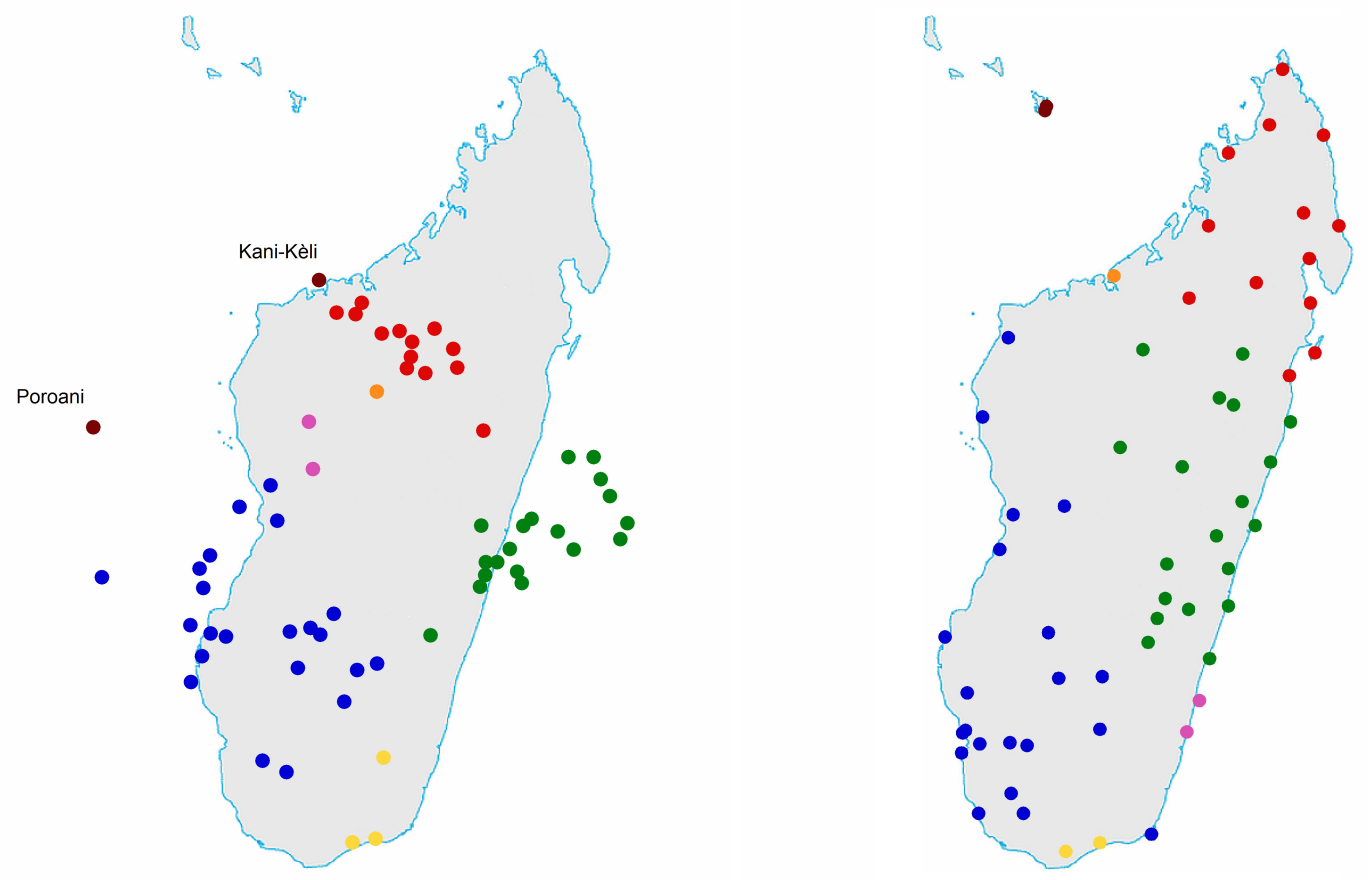

Figure 3: At the left the chart of Madagascar+Mayotte reconstructed by lexical input only, at the right the "true" chart where towns/villages are located according to geography (geodesic coordinates). The brown spots correspond to the two Mayotte varieties, all the other colors to the continental varieties. A yellow spot and a blue spot seem missing in the right chart, this is because both in Toliara and in Morondava two different varieties coexist. The Kimaore (KaniKéli) variety is lexically very close to the red cloud of the Northern dialects (left chart) and especially to the Ambanja Sakalava spoken in the Nosy Be region. The Kiantalaotsy (Poroani) is lexically close (but not very close) to the blue South-Western dialects, especially tto South-Western Sakalava varieties, however, its distance from the red Northern dialects is not much larger. It should be noted that Kibosy Kiantalaotsy does not appear to be particularly akin to Mahajanga Sakalava.

strategy only uses genealogical distances as input, no hints come from the "real" geography, i.e., no hints come from the geodesic coordinates of the towns/villages.

We first apply the method to the 60 continental varieties exactly as it is described in [1], and we end up with a set of 60 optimal bi-dimensional coordinates (lexical positions) $\overline{\mathbf{x}}_{1}, \overline{\mathbf{x}}_{2}, \ldots . ., \overline{\mathbf{x}}_{60}$ over the lexical plane. In order to render easier the visual comparison between the lexical positions (Fig. 3, left) and the geodesic coordinates (Fig. 3, right), we overturn, rotate, translate and zoom the lexical plane before superposing it to the Malagasy chart (Fig. 3, left). This operation only has the goal to make more intuitive the interpretation of our outcome, but in no way it is necessary.

In brief, the colored spots in the left chart of Fig. 3 correspond to the lexical positions, while the spots in the right chart (the "true" chart) correspond to the towns/villages geodesic coordinates. Almost all the spots in both charts refer to the continental varieties, with the exception of the brown ones which refer to the two dialects of Mayotte. Given that the lexical geography (or reconstructed geography) of continental varieties at the left of Fig. 3 is obtained only from vocabularies, the correspondence with the real geography at the right of Fig. 3 is surprising.

We still have to determine the position of the brown spots in the left chart of Fig. 3 i.e., the optimal lexical positions $\overline{\boldsymbol{\xi}}_{1}$ and $\overline{\boldsymbol{\xi}}_{2}$ of the two Mayotte dialects. We start by defining a cost function $S$ as 


$$
S\left(\boldsymbol{\xi}_{1}, \boldsymbol{\xi}_{2}\right)=S_{1}\left(\boldsymbol{\xi}_{1}\right)+S_{2}\left(\boldsymbol{\xi}_{2}\right)=\frac{1}{N} \sum_{\alpha=1}^{N}|T(1, \alpha)-| \boldsymbol{\xi}_{1}-\overline{\mathbf{x}}_{\alpha}||+\frac{1}{N} \sum_{\alpha=1}^{N}|T(2, \alpha)-| \boldsymbol{\xi}_{2}-\overline{\mathbf{x}}_{\alpha}||,
$$

where the sixty $T(1, \alpha)$ and the sixty $T(2, \alpha)$ are respectively the genealogical distances of Kimaore and Kiantalaotsy from the sixty continental varieties and were both the sums go over the $N=60$ continental varieties.

The $60+60$ quantities $|T(1, \alpha)-| \boldsymbol{\xi}_{1}-\overline{\mathbf{x}}_{\alpha}||$ and $|T(2, \alpha)-| \boldsymbol{\xi}_{2}-\overline{\mathbf{x}}_{\alpha}||$ measure the absolute difference between the euclidean distances on the lexical plane and the corresponding genealogical distances. The cost function $S$, which depends on the still undetermined lexical position $\boldsymbol{\xi}_{1}$ and $\boldsymbol{\xi}_{2}$, is the sum of all these differences.

The best lexical positions $\overline{\boldsymbol{\xi}}_{1}$ and $\overline{\boldsymbol{\xi}}_{2}$ are those which minimize $S$ (notice that minimization can be computed independently for the two bi-dimensional variables because of the sum structure $\left.S\left(\boldsymbol{\xi}_{1}, \boldsymbol{\xi}_{2}\right)=S_{1}\left(\boldsymbol{\xi}_{1}\right)+S_{2}\left(\boldsymbol{\xi}_{2}\right)\right)$. At the end of all the procedure, in addition to the sixty lexical positions $\overline{\mathbf{x}}_{1}, \overline{\mathbf{x}}_{2}, \ldots . ., \overline{\mathbf{x}}_{60}$ of the continental varieties, we also have the two lexical positions $\overline{\boldsymbol{\xi}}_{1}$ and $\overline{\boldsymbol{\xi}}_{2}$ of the Mayotte varieties.

As it can be appreciated in Fig. 3 (left), the lexical positions of the Kibosy dialects (brown spots) are very far from Mayotte. Kimaore is very close to the red cloud of the Northern Malagasy dialects, confirming the strict lexical link, while Kiantalaotsy is closer to the South-Western continental dialects. However, the distance of Kiantalaotsy from the South-Western dialects is not so small and the distance from the Northern dialects is not much larger. The almost equidistant lexical placing of Kiantalaotsy and its relative isolation (in the middle of the ocean) is compatible with a multiple origin of the dialect. Another possible explanation is that Kiantalaotsy diverged from the continental dialects a long time ago so that no very close relatives can be found in Madagascar. We find this explanation less plausible because of the extremely tight population bottleneck between the XVIIIth and the XIXth century,

Once again, as it can be appreciated in Fig. 3 (left), Kibosy Kiantalaotsy does not appear to be particularly akin to Mahajanga Sakalava. In sum, all the findings of the previous section are confirmed by this second analisys.

\section{Conclusions and outlook}

Mayotte is divided into two linguistic communities, The majority speaks Shimaore, which is one of the varieties of Comorian, a Bantu language which also includes the dialects of the other three Comorian islands. A minority, roughly one third of the population, speaks Kibosy (Kibushi in French/Bantu spelling) which means Malagasy.

Malagasy speakers do not occupy a part of the island but they live in about twenty villages scattered all around, especially in the East. The Malagasy variety named Kibosy Kiantalaotsy is only spoken in Poroani and Ouangani, while Kibosy Kimaore, a second well distinct Malagasy variety, is spoken in all the other villages.

It is widely believed that there is a strong affinity between Kibosy Kimaore and the Northern varieties of Malagasy (expecially those spoken in the Nosy Be region) and it is also widely believed that there is an even stronger affinity between Kibosy Kiantalaotsy and the Mahajanga Sakalava variety. While we clearly confirm the position of Kimaore, we find that Kiantalaotsy is closer to the South-Western Sakalava dialects with respect to the Mahajanga Sakalava variety. Moreover, Kibosy Kiantalaotsy, at variance with Kibosy Kimaore, shows to be relatively isolated from all the dialects spoken in Madagascar and it also shows a certain lexical equidistance from a number of West coast dialects. This may indicate multiple contributions to the genesis of this variety, which is not a weird phenomenon when dialects and not languages are involved.

Less likely, the lexical position of Kiantalaotsy could be due to an ancient branching from the Malagasy main stream. To make a choice between these two possible alternatives not only it is necessary a better understanding of the nature of the contact between Comorian Bantu and Kibosy languages [28] but, from our point of view, it is also necessary to quantify the extent of the horizontal transfer.

\section{Acknowledgments}

We are grateful to the Professors Heriniaina Andry Raboanary, Toky Hajatiana Raboanary, Julien Amédée Raboanary, Mara Edouard Remanevy, Dimby Vaovolo, Barthélemy Manjakahery and Marius Mandimbitafika Sambizafy for their invaluable advice concerning the varieties spoken in Madagascar and to Professors Mlaili Condro, Linda Rasomanana and Lina Hijab for data supply concerning the varieties spoken in Mayotte. 
We are also grateful to Alexander Adelaar, Murray Cox, Filippo Petroni, Dimitri Volchenkov and Soren Wichmann for a critical reading of the manuscript, suggestions and criticism.

The research has benefited of the logistical support of the Institut Supérieur Polytechnique de Madagascar (ISPM) and of the invaluable assistance of its teachers and students.

\section{Supporting Information}

Dataset 1: Kibosy Kimaore and Kibosy Kiantalaotsy Swadesh lists. The complete data-set of the 60 Swadesh lists for the Madagascar varieties can be found in the Supporting Information in [1].

Table 1: Ethnicities, towns and coordinates. The name of the $60+2$ dialects (ethnicities), the name of the towns/villages where dialects were collected together with their geodesic coordinates.

\section{References}

[1] M. Serva and M. Pasquini, Dialects of Madagascar. PLoS ONE 5(10), e0240170, (2020).

[2] P. Ottino, Madagascar, les Comores et le Sud-Ouest de l'Océan Indien. Antananarivo: Université de Madagascar, Publications du Centre d'Anthropologie Culturelle et Sociale, (1974).

[3] O. C. Dahl, Migration from Kalimantan to Madagascar. Oslo, Norway, Norwegian University Press. Institute for Comparative Research in Human Culture, (1991).

[4] A. Adelaar, Towards an integrated theory about the Indonesian migrations to Madagascar. In Ancient human migrations: a multidisciplinary approach, Salt Lake City: University of Utah Press, P. N. Peregrine, I. Peiros and M. Feldman editors, 149-172, (2009).

[5] Ph. Beaujard, The first migrants to Madagascar and their introduction of plants: linguistic and ethnological evidence. Azania: The Journal of the British Institute of History and Archaeology in East Africa, 46, 169189, (2011).

[6] A. Adelaar, Malagasy Phonological History and Bantu Influence. Oceanic Linguistics 51, 123-159, (2012)

[7] M. Serva, F. Petroni, D. Volchenkov \& S. Wichmann, Malagasy dialects and the peopling of Madagascar. Journal of the Royal Society Interface 9, 54-67, (2012).

[8] M. Serva, The settlement of Madagascar: what dialects and languages can tell us. PLoS ONE 7(2), e30666, (2012).

[9] R. Blust, The Austronesian Homeland and Dispersal. Annual Review of Linguistics, 5, 417-434, (2019).

[10] M. E. Hurles, B. C. Sykes, M. A. Jobling, and P. Forster, The dual origin of the Malagasy in Island SouthEast Asia and East Africa: Evidence from maternal and paternal lineages. American Journal of Human Genetics 76, 894-901, (2005).

[11] H. Soodyall, T. Jenkins and M. Stoneking, Polynesian mtDNA in the Malagasy. Nature Genetics 10, 377-378 (1995).

[12] S. Tofanelli, S. Bertoncini, L. Castrí, D. Luiselli, F. Calafell, G. Donati and G. Paoli, On the origins and admixture of Malagasy: New evidence from high-resolution analyses of paternal and maternal lineages. Molecular Biology and Evolution 26, 2109-2124, (2009).

[13] M. P. Cox, M. G. Nelson, M. K. Tumonggor, F-X. Ricaut and H. Sudoyo, A small cohort of Island SouthEast Asian women founded Madagascar Proceedings of the Royal Society B 279, 2761-2768, (2012). 
[14] P. Kusuma, M. P. Cox, D. Pierron, H. Razafindrazaka, N. Brucato, L. Tonasso, H. L. Suryadi, T. Letellier, H. Sudoyo and F.-X. Ricaut, Mitochondrial DNA and the Y chromosome suggest the settlement of Madagascar by Indonesian sea nomad populations. BMC Genomics 16, 191 (2015).

[15] N. Brucato, P. Kusuma, M. P. Cox, D. Pierron, G. A. Purnomo, A. Adelaar, T. Kivisild, T. Letellier, H. Sudoyo and F.-X. Ricaut, Malagasy Genetic Ancestry Comes from an Historical Malay Trading Post in SouthEast Borneo. Molecular Biology and Evolution 33, 2396-2400, (2016).

[16] N. Brucato, V. Fernandes, P. Kusuma, V. Černý, C. J. Mulligan, P. Soares, T. Rito, C. Besse, A. Boland, J.-F. Deleuze, M. P. Cox, H. Sudoyo, M. Stoneking, L. Pereira and F.-X. Ricaut, Evidence of Austronesian Genetic Lineages in East Africa and South Arabia: Complex Dispersal from Madagascar and SouthEast Asia. Genome Biology and Evolution 11, 748-758, (2019).

[17] A. Adelaar, A linguist's perspective on the settlement history of Madagascar. NUSA 61, 69-88, (2017).

[18] A. Crowther, L. Leilani, R. Helm, M. Horton, C. Shipton, H.T. Wright, S. Walshaw, M. Pawlowicz, C. Radimilahy, K. Douka, L. Picornell-Gelabert, D.Q. Fuller and N. L. Boivin, Ancient crops provide first archaeological signature of the westward Austronesian expansion. Proceedings of the National Academy of Sciences of the United States of America 113, 6635-6640, (2016).

[19] N. J. Gueunier, Le dialecte malgache de Mayotte (Comores): une discussion dialectologique et sociolinguistique. Faits de Langues, 23, 397-420, (2009).

[20] N. J. Gueunier, Dictionnaire du dialecte malgache de Mayotte (Comores). Édition revue et corrigée published by KomEDIT, (2016).

[21] Jules-Sébastien-César Dumont d'Urville Sur les iles du Grand Ocean. Bulletin de la Société de Gèographie 17, 1-21, (1832).

[22] M. Swadesh, Lexicostatistic dating of prehistoric ethnic contacts. Proceedings of the American Philosophical Society 96, 452-463, (1952).

[23] M. Serva and F. Petroni, Indo-European languages tree by Levenshtein distance. EuroPhysics Letters 81, $68005,(2008)$.

[24] M. Pasquini and M. Serva, Horizontal transfers are a primary aspect of languages evolution. Europhysics Letters 125, 38002, (2019).

[25] M. Pasquini and M. Serva, Stability of meanings versus rate of replacement of words: an experimental test. Journal of Quantitative Linguistics, (on line 6 Aug 2019).

[26] C. Allibert, L'archipel des Comores et son histoire ancienne. Essai de mise en perspective des chroniques, de la tradition orale et des typologies de céramiques locales et d'importation. Afriques 06, L'Afrique orientale et l'océan Indien : connexions, réseaux d'échanges et globalisation, (2015).

[27] M. Serva, D. Vergni, D. Volchenkov \& A. Vulpiani Recovering geography from a matrix of genetic distances. Europhysics Letters 118, 48003, (2017).

[28] A. Adelaar, Language contact in Africa. To appear in The Oxford Guide to the Malayo-Polynesian languages of South East Asia, A. Adelaar and A. Schapper editors. Oxford Guides to the World's Languages, Oxford: OUP, (2021). 